\title{
Reflets
}

Revue ontaroise d'intervention sociale et communautaire

\section{Le défi des pratiques d'intervention interculturelle dans le champ de la santé sexuelle}

\section{Claire Parrot}

\section{Volume 4, numéro 1, printemps 1998}

Intervention en contextes minoritaires

URI : https://id.erudit.org/iderudit/026200ar

DOI : https://doi.org/10.7202/026200ar

Aller au sommaire du numéro

Éditeur(s)

Reflets : Revue ontaroise d'intervention sociale et communautaire

ISSN

1203-4576 (imprimé)

1712-8498 (numérique)

Découvrir la revue

Citer cet article

Parrot, C. (1998). Le défi des pratiques d'intervention interculturelle dans le champ de la santé sexuelle. Reflets, 4(1), 128-139.

https://doi.org/10.7202/026200ar
Résumé de l'article

Un quart des 10 millions de personnes qui habitent en Ontario sont des membres d'uneminorité raciale, des immigrantes et immigrants récents ou des personnes réfugiées. Cela veutdire que parmi ces personnes, il y a plus d'un million de jeunes filles et de femmes qui ont unproblème commun : elles ne reçoivent pas les services de santé qu'elles méritent. (ministère dela Santé de l’Ontario 1993).
Tous droits réservés (C) Reflets : Revue ontaroise d'intervention sociale et communautaire, 1998
Ce document est protégé par la loi sur le droit d'auteur. L'utilisation des services d’Érudit (y compris la reproduction) est assujettie à sa politique d'utilisation que vous pouvez consulter en ligne.

https://apropos.erudit.org/fr/usagers/politique-dutilisation/ 


\section{Le défi des pratiques d'intervention interculturelle dans le champ de la santé sexuelle}

Un quart des 10 millions de personnes qui habitent en Ontario sont des membres d'une minorité raciale, des immigrantes et immigrants récents ou des personnes réfugiées. Cela veut dire que parmi ces personnes, il $y$ a plus d'un million de jeunes filles et de femmes qui ont un problème commun: elles ne reçoivent pas les services de santé qu'elles méritent. (ministère de la Santé de l'Ontario 1993).

\section{Claire Parrot \\ Infirmière autorisée et consultante en matière de santé sexuelle, Toronto}

Même lorsqu'on a vécu en Ontario depuis plusieurs années, qu'on a baigné dans une culture plutôt conciliante, qu'on a vécu une enfance choyée, qu'on s'exprime très facilement en anglais, la santé sexuelle demeure un sujet à controverses. Or qu'en est-il des femmes nouvellement arrivées au pays, provenant de cultures différentes, parlant à peine l'anglais ou pas du tout et qui ont de nombreux obstacles à surmonter? Où leur santé sexuelle?

C'est un défi de taille pour la professionnelle de la santé qui a une certaine expertise dans le domaine mais tout à apprendre de l'univers privé de sa cliente immigrante. Dans ce texte, je définis le terme «intervenante» comme «une personne qui opère, qui joue un rôle dans une relation d'aide».

Je tenterai de cerner les difficultés, les peines et gratifications de l'intervenante francophone qui malgré tout, a encore souvenance de sa culture française judéo-chrétienne et qui a à coeur 
de rendre un service totalement adapté à la personnalité de sa cliente. Le fait de converser dans la même langue ne rend pas la situation immuable, un écart plus ou moins grand séparant la professionnelle de la cliente... il s'agit de le réduire.

Ces défis se dessinent tantôt sous forme d'émotions, de politiques, d'éducation, d'apprentissage, tantôt sous forme de préjugés, de questions philosophiques, de relation d'aide, mais surtout, ils rendent nécessaire un grand respect pour toutes ces femmes qui ne demandent qu'à apprendre.

Mais qu'est-ce au juste que la «santé sexuelle»? D'après l'Organisation mondiale de la santé, la santé sexuelle c'est

...l'intégration des aspects somatiques, intellectuels, affectifs et sociaux de l'être sexué, réalisée selon des modalités épanouissantes qui valorisent la personnalité, la communication et l'amour». "[La santé sexuelle suppose]... la réunion de trois conditions fondamentales:...être capable de jouir, avec pleine maitrise, d'un comportement sexuel et reproducteur en harmonie avec une éthique sociale et personnelle. (Santé Canada 1986: 6)

L'éducation en santé sexuelle comporte plusieurs défis qui souvent se présentent à l'insu des intervenants. Les différents aspects de la vie d'une personne reflètent sa santé sexuelle ainsi que les moyens qu'elle possède pour arriver à améliorer la situation présente. Il s'avère très ardu mais combien enrichissant de relever ces défis... Lorsque la notion d'éducation est bien perçue, que l'éducatrice y tient un rôle majeur mais non complaisant, les acteurs de part et d'autre forment une équipe gagnante. En effet, des experts en ce domaine affirment :

La composante pédagogique de la promotion de la santé, c'est-à-dire l'éducation en matière de santé, fournit aux individus les connaissances, les aptitudes et la sensibilisation critique qui leur permettront de faire des choix volontaires et éclairés au sujet des changements personnels ou sociaux à effectuer pour améliorer leur santé. (Santé Canada 1986: 6) 
Situé au centre de Toronto, The Bay Centre for Birth Control se définit comme un «Centre pour la santé reproductive et sexuelle des femmes». La clinique fait partie du Regional Women's Health Centre lequel est affilié à l'hôpital Women's College de Toronto. The Bay Centre for Birth Control offre une gamme de services visant à améliorer la santé reproductive des femmes; de plus, il est l'hôte pour bon nombre d'étudiants-tes en médecine, sciences infirmières et sciences sociales qui désirent parfaire leurs connaissances dans le domaine de la contraception. Le public a aussi accès à une bibliothèque de prêt, The Women's Health Resource Centre qui regorge d'une grande variété de matériel éducatif tel livres, articles, revues et textes de références.

Infirmières, médecins et la travailleuse sociale possèdent l'expertise nécessaire pour amener la cliente à effectuer un choix éclairé. Parmi les nombreuses options offertes au Centre, on retrouve d'une part, des renseignements généraux, sessions de counselling et services médicaux reliés aux méthodes de contraception, les plus connues et les plus récentes; d'autre part, on s'efforce constamment de percevoir la femme comme une personne à part entière, dont les besoins immédiats s'insèrent à l'intérieur d'une optique beaucoup plus grande. Il est maintenant reconnu que la santé reproductive s'enchâsse dans le domaine plus complexe mais non moins intéressant de la santé sexuelle.

Depuis 1990, la clinique The Bay Centre for Birth Control a reçu 526 femmes dont la langue maternelle est le français; 371 d'entre elles viennent du Canada, le reste c'est-à-dire 155, sont originaires de 13 pays d'Afrique. ${ }^{1}$ Les immigrantes francophones en provenance des pays d'Afrique sont originaires d'Éthiopie, de la République démocratique du Congo (exemple Zaïre), du Mali, du Burundi, du Rwanda, de l'Égypte, du Libéria, de la Zambie, de la Côte d'Ivoire et de Madagascar pour n'en nommer que quelques-uns... On peut avancer que la culture et le vécu de ces femmes s'allient parfois difficilement avec certains impératifs de la culture nord-américaine. Elles arrivent chez-nous avec un bagage souvent riche tant au point de vue culturel qu'intellectuel, mais hélas, elles apportent aussi un passé empreint d'horreurs et 
lourd de peines. Je cite ici Rita Parihk, chercheure féministe et militante:

L'histoire du monde, c'est l'histoire des populations qui se déplacent et qui partent à la recherche de terres en quête de richesses et de ressources, de sécurité et de nouvelles possibilités. C'est toutefois du tiers monde que provient maintenant la plus grande partie des courants migratoires. Ainsi, le Zaïre et le Kénya sont par exemple, des pays qui répondent aux besoins de millions de réfugiés africains qui fuient la famine ou la tyrannie régnant dans leur pays (1996: 21-22).

Infirmière de profession, je suis la seule intervenante du centre à parler français couramment. Au cours des ans, $\mathrm{j}$ 'ai appris par mon expérience personnelle et professionnelle, qu'il était profitable «d'élargir ses horizons» pour être mieux outillée. Le «résautage» (établir des contacts avec la communauté ou avec des individus) est un de ces outils. Bien souvent donc, les clientes francophones qui désirent me rencontrer connaissent déjà mon nom; il leur a été donné soit par une amie ou une généraliste, soit par des centres de santé où on accueille grand nombre de femmes immigrantes.

Lorsque je m'entretiens avec des clientes africaines, elles me confient à maintes reprises qu'elles ont dû fuir leur pays natal pour les raisons qu'on a évoquées plus tôt. Au premier abord, c'est-à-dire lorsque je prononce les premiers mots de bienvenue, je peux me rendre compte que la requérante est soulagée et me répond d'un sourire qui en dit long... Sinon, c'est la surprise totale suivie d'un assentiment un peu timide. La première étape se fait sans heurt. La plupart du temps, quelle que soit la raison de sa visite, la femme immigrante francophone me laissera savoir verbalement ou non, qu'elle se sent déjà rassurée puisque nous parlons la même langue et que je pourrai répondre plus facilement à ses besoins. Bien sûr, $\mathrm{j}$ 'aurai au préalable pris connaissance de ses coordonnées : raison de la visite, pays d'origine, âge, etc., pour ainsi être en mesure de me familiariser avec ma cliente. Dès lors, un climat de confiance s'installe peu à peu. Il y a bien sûr plusieurs façons d'intervenir auprès de la cliente; je crois cependant qu'une 
bonne réceptivité et un désir sincère d'aider l'autre aboutissent à de bons résultats. Psychologie oui, mais sociologie aussi. Les deux à l'unisson me permettent de maintenir cette confiance. Je m'assure de lui expliquer clairement quel est mon rôle en tant qu'infirmière-éducatrice: que mes connaissances médicales pourront l'aider à voir plus clair dans ce qui l'inquiète du côté physique; que mon expertise dans la santé reproductive des femmes, biologique et émotive, pourrait l'amener vers un choix qui sera le sien et qui lui donnera probablement une certaine sérénité.

Le choix de la cliente, voilà un élément très important. Je répète souvent: "peu importe le choix que tu feras, ce sera le tien». Il est trop facile de donner à quelqu'un l'impression qu'on la «sauvera» en lui disant quoi faire ou en lui montrant la direction à prendre. Il y a des situations si lourdes de conséquences que l'intervenante ne peut s'approprier les sentiments de la cliente, lesquels se répercuteront dans un avenir plus ou moins rapproché. Je nomme la culpabilité, la rage, la vengeance, la peur du partenaire, etc. Donc, dans un langage simple mais précis et ferme,j'explique à cette femme qu'elle doit prendre en charge sa santé.

Il va sans dire qu'à n'importe quel moment de l'entretien, à point nommé, la requérante immigrante a toujours le loisir d'arrêter l'interaction ou de changer de direction; si par exemple elle se sent trop fragile, si elle veut pleurer un bon coup ou si elle

«Il est parfois tentant de se trouver en situation de pouvoir lorsqu'on sait qu'on peut aider une personne démunie à plusieurs points de vue.» demande la présence de son partenaire, de son amie. Il est parfois tentant de se trouver en situation de pouvoir lorsqu'on sait qu'on peut aider une personne démunie à plusieurs points de vue. Bien qu'en même temps, les résultats escomptés soient souvent valorisants, il faut prendre garde de ne pas verser dans la complaisance. Voilà pourquoi je m'efforce aussi souvent que s'y prête la situation, de lui montrer que moi aussi j'apprends, que j'échange avec elle, que j'aime à découvrir de nouveaux vocables français, des coutumes qui m'étaient inconnues; je m'y retrouve tellement plus enrichie. Il m'arrive de pleurer avec elle, elle sait que c'est de l'empathie et non du misérabilisme, parce que son histoire me touche. De temps à autre, une main sur son épaule lui rappelle qu'elle n'est pas tout à fait seule. L'humour est sans contredit une arme très efficace pour alléger les moments les plus 
difficiles. J'essaie de l'employer souvent et je le conseille toujours aux étudiants qui viennent apprendre chez-nous.

La langue est l'un des moyens de communication le plus utilisé. Nancy Waxler-Morrison avance qu'

immigrer demande de s'adapter à un nouvelle société. Plusieurs immigrés doivent apprendre une nouvelle langue, se mettre à la recherche de supports variés, changer les habitudes alimentaires, etc. (1990: 6-7)

Elle souligne en effet les obstacles souvent rencontrés par les personne immigrantes: difficulté de s'exprimer en anglais, manque d'argent, difficulté à décoder la bureaucratie gouvernementale. Fait flagrant, cette lacune de la langue augmente indéniablement la frustration, ce qui rend encore plus difficile l'accès des services de santé dont les femmes ont besoin et, trop souvent elles se retrouvent déçues de ces services.

Janice Irvine, figure de proue américaine dans le domaine de l'éducation en santé sexuelle, a étudié plusieurs questions au niveau de la langue, des stratégies conversationnelles et des structures de communication, et ce dans le contexte des approches interculturelles. Elle met l'accent sur la subtilité de la langue lorsqu'il s'agit de communiquer un message concernant l'éducation sexuelle. En septembre 1994, le Caire était l'hôte d'un colloque parrainé par «United Nations on Population and Development». Quand vint le temps de compiler le résultat des discussions et de les traduire dans chacune des langues des pays participants, ce fut la tour de Babel! On n'arrivait pas à s'entendre sur la traduction; des termes comme «éducation sexuelle et santé

"Ces exemples de défis linguistiques et communicationels en termes de santé sexuelle démontrent assez bien la complexité du débat de la langue comme outil idéal pour s'exprimer.» sexuelle», par exemple, étaient extrêmement difficiles à interpréter dans la langue arabe parce que trop offensants. Il en était de même pour les pays francophones qui clamaient qu'il était presque impossible de traduire l'expression «empowerment for women». Ces derniers ont finalement opté pour «renforcement du pouvoir d'action des femmes» (Irvine 1995: 109).

Ces exemples de défis linguistiques et de communication en termes de santé sexuelle démontrent assez bien la complexité du 
débat de la langue comme outil idéal pour s'exprimer. Cela nous démontre aussi que «le terrain est parfois très glissant». Tout compte fait, Irvine résume d'une façon remarquable en disant :

Il n'y a pas de recette magique lorsqu'on parle de défis culturels dans le langage et la communication. La réalité de ces modèles et une bonne connaissance interculturelle faciliteront la tâche de l'éducateur qui se verra capable d'employer l'approche spécifique pour la clientèle visée. (1995: 123)

Toute personne ayant reçu une formation professionnelle doit se soumettre à un certain code d'éthique. L'Ordre des infirmiers et infirmières de l'Ontario est le corps professionnel qui gère les compétences de ses membres. Le $1^{\text {er }}$ janvier 1997, l'Ordre des infirmières et des infirmiers de l'Ontario initiait, le Programme d'assurance de la qualité (PAQ). Force est d'admettre que la vaste majorité des professions sont bombardées de tous côtés avec les courants politiques, les conflits sociaux et les droits que tout un chacun revendique à qui mieux mieux. Les infirmiers et les infirmières des années 90 n'y font pas exception. Ces professionnels ont vu de nombreux changements s'opérer dans leur domaine, tant au niveau technologique qu'au niveau humanitaire et plus précisément dans la relation d'aide. Dans un milieu médical où la clientèle comprend en grande partie des femmes démunies, parfois désavantagées physiquement ou intellectuellement et, par surcroît, réfugiées, l'intervenante a la tâche de s'adapter si elle veut survivre, tout en ayant la satisfaction d'être professionnelle avec tout ce que cela implique.

Le nursing interculturel se définit ainsi :

Un domaine du nursing dans lequel l'infirmier(ère) transcende un ethnocentrisme et pratique sa profession à l'intérieur d'une autre sphère culturelle. Le nursing intercuturel fait partie intégrante de toutes pratiques de nursing parce que sa théorie et son courant présents n'ont pas de frontières culturelles et parce que les besoins de chaque personne doivent être perçus d'une façon individuelle». ${ }^{2}$ 
"...les situations sont toujours différentes parce que les clientes le sont, l'approche éducative par contre est semblable, bien que la professionnelle doive incessamment repenser ses valeurs, ses croyances et sa place dans la société.»
Par la force des choses et fort heureusement, la professionnelle de la santé acquiert une foule d'aptitudes, lesquelles l'amènent à s'adapter au besoin, selon la situation donnée. Dans le cadre et l'atmosphère d'une clinique telle que The Bay Centre for Birth Control, ces adaptations se font fréquemment. Cette même professionnelle a donc plusieurs cordes à son arc; mais sait-elle toujours quand et comment les utiliser? La clinique favorisant l'enseignement de plusieurs disciplines, le personnel est donc plus à l'écoute des besoins des uns et des autres. Il va sans dire que les situations sont toujours différentes parce que les clientes le sont; l'approche éducative par contre est semblable, bien que la professionnelle doive incessamment repenser ses valeurs, ses croyances et sa place dans la société.

Pourquoi? Parce que les situations évoquées par les clientes suscitent le doute, la condescendance et voire l'opprobre; elles sont toutes plus réelles les unes que les autres. Combien de fois lit-on atrocités et aberrations qui font la une des grands quotidiens de la ville? Ces «faits désolants», on les voit en chair et en os dans les centres de santé pour femmes... La professionnelle doit donc s'armer de courage et d'intégrité, elle doit faire confiance à son intuition. Sa vie professionnelle se trouve sans cesse confrontée à des éléments qui peuvent refléter une situation personnelle, passée ou présente.

Une femme immigrante, ne parlant peu ou pas l'anglais, se présente avec une requête qui en masque souvent plusieurs autres: elle est enceinte et ne sait trop quoi faire: veut-elle interrompre la grossesse? Sait-elle qu'elle a le droit de faire adopter son enfant si elle décidait de mener la grossesse à terme? Est-elle est victime d'inceste ou d'agression sexuelle? Son partenaire abuse-t-il d'elle, que ce soit de façon verbale, physique ou émotive? Y a-t-il quelqu'un qui l'oblige à interrompre sa grossesse? Comment la recevra-t-on? Saura-t-on la comprendre? Est-ce qu'on arrivera à décoder ses craintes, ses espoirs? La couleur de sa peau sera-t-elle un obstacle de plus? Cette personne a le droit de recevoir un service à la mesure de ses besoins et de sa situation.

L'éducatrice en santé sexuelle peut et doit, d'une façon constante, accueillir la femme immigrante en lui offrant une 
attitude positive qui facilitera son cheminement vers un mieuxêtre. Un peu plus haut, nous évoquions certains des critères idéaux pour aborder le délicat sujet de la sexualité: d'entrée de jeu, on sait que ce chapitre de la santé porte à confusion et demande d'avoir acquis de l'expérience dans le domaine. Irvine encore une fois avance: «La culture se décrit comme un ensemble de vues créées historiquement, de règles et de pratiques par lesquelles groupes et sousgroupes s'organisent. La culture devient alors une sorte de guide qui nous aide à négocier les aspects de notre vie quotidienne..» (1995:25). Voilà pourquoi la tâche s'amplifie lorsque nous avons affaire à une culture autre que la nôtre et qu'il nous faut transmettre des messages qui peuvent être tout à fait tabous pour certaines clientes et moins pour d'autres.

Plusieurs études ont déjà démontré que les gens apprennent différemment; l'apprentissage est une faculté qui se développe selon de nombreux facteurs. Il va de soi que l'éducatrice doit tenir compte de la personne à qui elle enseigne: l'âge, le niveau d'instruction, la langue, la capacité intellectuelle, etc. Richard Brislin, psychologue de formation, explique dans ses écrits que «dans la communication, la nécessité de base est de comprendre ce que veulent dire les moindres sons émis par les autres... Dans un contexte interculturel, il est beaucoup plus ardu d'obtenir une bonne compréhension puisque les gens apportent différents vécus, besoins et valeurs au procédé de communication» (1981:65).

Réexaminons maintenant la situation des femmes francophones immigrantes dans le contexte de la santé sexuelle. En principe, elles se présentent à un centre de santé pour femmes, en l'occurence le Bay Centre for Birth Control, pour les raisons qui sont sensiblement les mêmes que les autres clientes. Là où il y a variante, c'est dans la manière d'intercéder auprès de ces dernières; gardons en tête leur cheminement passé et leurs besoins immédiats. La plupart des pays du tiers-monde sont encore empreints d'une forte influence patriarcale bien souvent mêlée à une religion peu tolérante. Il devient alors facile de juger leur façon de voir la vie, leur acceptation de rituels qui nous paraissent comme une forme de torture.

Prenons l'exemple de la mutilation des organes génitaux de la femme. Jusqu'à ce jour, 114 millions de femmes auraient subi 
«Plusieurs de mes clientes africaines m'ont cependant affirmé qu'elles préféraient revenir me voir, ou aller rencontrer quelqu'un d'autre qu'on leur aura recommandé au préalable et qui aura une culture différente de la sienne." une des formes de la mutilation. Une fois l'horreur dissipée, la compassion installée, il reste tant à faire... Peut-on se renseigner auprès de la cliente sur son mode de vie, ses valeurs, ses peurs, etc.? Bien sûr.Y a-t-il possibilité d'assurer un suivi avec une autre intervenante dans le milieu hospitalier ou la communauté? Absolument. Plusieurs de mes clientes africaines m'ont cependant affirmé qu'elles préféraient revenir me voir, ou aller rencontrer quelqu'un d'autre qu'on leur aura recommandé au préalable et qui aura une culture différente de la sienne. La raison: elles ont peur que s'ébruitent certaines choses... Le respect de la cliente en tout temps est de rigueur.

Des collègues et connaissances qui oeuvrent dans la recherche ou sur le «terrain» de l'éducation interculturelle me disaient que les femmes noires francophones ressentaient une sorte de condescendance de la part des intervenants de la santé; que cellesci ont l'impression que les femmes immigrantes sont toujours sous un quelconque joug. Il est impératif que les centres de santé pour femmes se mettent au diapason des vécus culturels qu'apportent les courants migratoires. Janice Irvine nous incite à toujours regarder d'un oeil vigilant le champ de l'éducation sexuelle dans un contexte intercuturel, de peur de se perdre en d'inutiles conjectures. Une bonne dose de réalisme et une motivation constante sauront aider les éducatrices; elles voudront de plus en plus apprendre à connaitre d'autres cultures. Bref, elles pourront faire leur propre recherche, écouter la femme immigrante et interpréter le plus fidèlement possible ce qu'elle aura à raconter... Il s'agit donc d'ajuster son tir et d'utiliser l'approche la plus adéquate possible (1995: 123).

\section{De nombreux obstacles restent cependant à franchir}

Au mois d'août 1993, le ministère de la Santé de l'Ontario publiait un document concernant les besoins des immigrantes, des régugiées et des femmes des minorités raciales. Quelques points tirés de la brochure appuient plusieurs aspects du présent article: 
"Ces femmes ont leur porte-parole; elles demandent entre autre que les personnes formées à l'étranger puissent travailler dans une profession de la santé sans avoir à traverser un océan de bureaucratie pour qu'on reconnaisse leurs compétences." la langue constitue le principal obstacle aux soins de santé des immigrantes; elles sont souvent dans l'impossibilité de décrire ce qui ne va pas; elles peuvent être découragées par les professionnels de la santé qui leur parlent avec hauteur en employant des mots très techniques; elles ne comprennent pas toujours qu'elles ont droit aux soins de santé subventionnés; elles ont l'impression de ne pas toujours comprendre ce que leur dit le médecin ou l'intervenante; elles ont du mal à se rendre chez le médecin ou à la clinique parce qu'elles n'ont pas les moyens de faire garder leurs enfants.

Les intervenantes en santé sexuelle détiennent le temps et les outils nécessaires pour entrer en relation d'aide avec leur clientes immigrantes. Encore faut-il la collaboration des personnes qui détiennent le pouvoir pour arriver à faire une différence remarquable. En même temps, il faut des solutions immédiates. Ces femmes ont leur porte-parole; elles demandent entre autres que les personnes formées à l'étranger puissent travailler dans une profession de la santé sans avoir à traverser un océan de bureaucratie pour qu'on reconnaisse leurs compétences.

Malgré ces durs défis, les éducateurs et les éducatrices en santé, de surcroît en santé sexuelle, peuvent donner le choix à leurs clientes en montrant beaucoup de franchise et une grande délicatesse. Michelle Laaroussi nous recommande«d'éviter le folklorisme ou le culturalisme réducteur, et de se situer comme nous l'avons fait, dans une position d'écoute et de découverte des femmes immigrantes» (1995:1,2). En effet, «aucun être humain ne peut être réduit à ce que nous voyons ou à ce que nous croyons voir. Chaque personne est infiniment plus grande, plus profonde que ce que notre étroitesse d'esprit peut discerner». (De Hennezel 1997: 19).

\section{Bibliographie}

BARRETT et al. (1994). Lignes directrices nationales pour l'éducation en matière de santé sexuelle, Ottawa, ministère des Approvisionnements et Services Canada, Cat H39 300/1994F.

BRISLIN, Richard W. (1981). Cross-Cultural Encounters Face-to-Face Interaction, Toronto, Pergamon Canada Ltd., 40-42, 65. 
BRISLIN, R., K. CULSNER, C. CHERRIE et M.YONG (1986). Intercultural Interactions, A Practical Guide, California, Sage Publication, 304-306.

BRISLIN, Richard W. (1990). Applied Cross-Cultural Psychology, California, Sage Publications,Inc., 9-11.

DE HENNEZEL, Marie (1995). La mort intime, Paris, Éditions Robert Laffont.

GOUDREAU, Hélène (1997). Femmes immigrantes Reconstruire un ailleurs, www.usherb.ca./ LIAISON/vol30/v30n18/IMMIGRANTE.html, compte rendu de «Femmes immigrantes à Sherbrooke: mode de vie et reconstruction identitaire" par Le Collectif de recherche sur les femmes et le changement (CRFEC), (1995), Sherbrooke, Liaison, vol. 30, no 18.

IRVINE, M. Janice (1995). Sexuality Education Across Cultures Working with Differences, San Francisco, California, Jossey-Bass Inc.

MINISTÈRE DE LA SANTÉ DE L'ONTARIO (1993). Les besoins des immigrantes, des réfugiées et des femmes des minorités raciales en matière de services de santé,Toronto, Rapport des consutlations communautaires, 1-11.

OFFICE DES AFFAIRES FRANCOPHONES et STATISTIQUE CANADA

GOUVERNEMENT DE L'ONTARIO (1991). Les femmes francophones en Ontario Profil statistique, Toronto, Queen's Park, 5-11.

PARIKH, Rita (1996). Au nom du développement, Ottawa, Inter Pares.

SANTÉ CANADA (1986). Lignes directrices nationales pour l'éducation en matière de santé sexuelle, Ottawa.

WAXLER-MORRISON, A.J., \& Richardson, E. (1990). Cross Cultural Caring a Handbook for Professionals in Western Canada, Vancouver, UBC Press, 6, 7.

\section{Notes}

1. Regional Women's Health Centre, Bay Centre for Birth Control

2. Traduction libre de Mosby's Medical, Nursing, and Allied Health Dictionary 